RAL Report

\title{
Simple Model for the Spin-Crisis
}

\author{
A A Anselm M Ryskin
}

March 1995 
DRAL is part of the Engineering and Physical

\section{Sciences Research Council}

The Engineering and Physical Sciences Research Council does not accept any responsibility for loss or damage arising from the use of information contained in any of its reports or in any communication about its tests or investigations 
RAL-95-030

March 1995

\title{
Simple Model for the Spin-Crisis
}

\author{
A.A. Anselm \\ Petersburg Nuclear Physics Institute \\ Gatchina, 188350 St. Petersburg, Russia \\ and \\ M. Ryskin ${ }^{1}$ \\ Rutherford Appleton Laboratory, \\ Chilton, Didcot OX11 0QX, England. \\ and \\ Petersburg Nuclear Physics Institute \\ Gatchina, 188350 St. Petersburg, Russia
}

\begin{abstract}
Starting from the Additive Quark SU(6) Model and taking into account pion, kaon and $\eta$ emission during the initial stage of evolution (from $q^{2} \sim m_{\text {quark }}^{2}$ to $q^{2} \sim 1 \mathrm{GeV}^{2}$ ) we convert the main fraction of the proton spin into the orbital momentum of pseudoscalar mesons. The results are in good agreement with the experimental data. We get $\int g_{1}(x) d x=0.152$ for proton and +0.007 for the $\Lambda$ hyperon (i.e. the quarks carry out only about $39 \%$ of the $\Lambda$ spin). In the same model we explain the violation of the Gottfried sum rule, $\int\left(F_{2}^{p}(x)-F_{2}^{n}(x)\right) d x=0.23$ due to charged meson exchanges at the beginning of the evolution. The Gribov-Lipatov relation between the polarized structure functions in DIS and $e^{+} e^{-}$-annihilation is discussed. It allows the possibility of studying the spin structure of $\Lambda$ hyperon produced through the decay of a jet originating from a polarized quark in $e^{+} e^{-}$annihilation or in DIS.
\end{abstract}

\footnotetext{
${ }^{1}$ This work was support in part, by a Soros Foundation Grant awarded by American Physical Society.
} 

The so-called "spin crisis" problem has generated a lot of publications after the EMC experiment [1] where the polarized quark structure function $g_{1}$ was measured for the first time. The value of the integral $\int g_{1}(x) d x=0.126 \pm 0.018$ turns out to be much less than the $\mathrm{SU}(6)$ prediction $\sim 0.28$. It means that less than half of the initial proton spin is carried by the quarks. One can find a detail review of the subject in ref. [2].

It is well known that due to the Adler-Bell-Jackiw anomaly [3] the singlet (with respect to the Havour symmetry $\left.S U(3)_{F}\right)$ axial current $J_{5 \mu}^{0}=\sum_{F} \bar{q} \gamma_{5} \gamma_{\mu} q$, which controls the total spin carried by the quarks, is not conserved. Nevertheless, within perturbative QCD this nonconservation is small and almost negligible. In the region of rather large $Q^{2} \gg 1 \mathrm{GeV}^{2}$ (where the QCD coupling $\alpha_{s} \ll 1$ ) the anomalous dimension $\gamma^{0}$ which describes the $Q^{2}$ dependence of the $J_{5 \mu}^{0}$ operator is proportional to the square of $\alpha_{s}[4]$

$$
\gamma_{0}=\frac{1}{2}\left(\frac{\alpha_{s}}{\pi}\right)^{2} n_{F}
$$

Thus the variation of the total quark spin

$$
J_{5 \mu}^{0}\left(Q^{2}\right)=J_{5 \mu}^{0}\left(Q_{0}^{2}\right) \exp \left[-\int_{Q_{0}^{2}}^{Q^{2}} \gamma^{0} \frac{d Q^{2}}{Q^{2}}\right]=J_{5 \mu}^{0}\left(Q_{0}^{2}\right) \exp \left[\frac{2 n_{F}}{b \pi}\left(\alpha_{s}\left(Q^{2}\right)-\alpha_{s}\left(Q_{0}^{2}\right)\right)\right]
$$

does not exceed $7 \%$ for $\alpha_{s}\left(Q_{0}^{2}\right) \leq 0.35$ (here we put the number of light flavours $n_{F}=3$ and the Gell-Mann-Low constant $b=11-2 / 3 n_{F}=9$ ).

However it is not excluded that the $J_{5 \mu}^{0}$-current non-conservation may reveal itself in the non-perturbative region of not too large $Q^{2} \leq 1 \mathrm{GeV}^{2}$. To study this problem we will use the effective QCD Lagrangian, which deals only with the lightest degrees of freedom: the constituent $u, d, s$-quarks and the octet of the pseudoscalar mesons $(\pi, K, \eta)$.

Our goal is to consider a model which is very close to the additive quark one, but starting from $\mathrm{SU}(6)$ we will follow the initial part of the structure function evolution. Due to the possibility of emitting the pseudoscalar mesons $(\pi$ or $K, \eta)$ the initial valence quark may flip its spin and thus reduce the sum of the quark chiralities $(\Delta \Sigma)$; the remaining part of the proton spin goes to the orbital momentum of the parton wave function. The probability of the pion (kaon) emission is given by the ratio $\frac{1}{16 \pi^{2}} \frac{M^{2}}{f_{\pi}^{2}}$ (where $M$ is the constituent quark mass). It is not large but within the low $q^{2}$ interval (before the pion form factor reveals itself and damps the contribution) it is enhanced by the $\log$ integration $\left.\int d q^{2} / q^{2}\right)$ and finally produces a noticeable effect which is almost enough to explain the spin crisis. 
An idea very close to this was discussed before in the framework of a slightly different Lagrangian[5] in the context of the Gottfried sum rule violation. The possibility of transferring part of the initial proton spin to the pions emitted by the parent nucleon was considered also in [6]. Thus our explanation of spin crisis is not a completely new one. The advantage is that we start from the simplest Lagrangian (see eq.(3)), which preserves all the symmetry relations (isotopic symmetry will be fulfilled, for example) and write an evolution equation which gives the possibility to study (and predict) the $x$-dependence of polarized quark structure functions, not just the contribution of quarks to the nucleon spin, $\Delta \Sigma$. It is also possible to consider different baryons such as the $\Lambda$-hyperon, not just the nucleons ${ }^{2}$.

In section 2 we recall the structure of the effective QCD Lagrangian. Then in section 3 we write the system of equations, which describes the evolution of the spin dependent structure functions in this case. The numerical results of the solution is discussed in section 4 .

In the conclusion (section 5) we show that the same model also succeeds in explaining the Gottfried sum rule violation (due to charged pion exchange). Also, since the model includes the $q^{2}$ evolution explicitly, it allows the possibility of the data of E142 [8] and SMC [9], which differ from each other, to be made consistent since they are measured at different values of $Q^{2}$. For the E142 data the $Q^{2}$ is rather small $\sim 2 \mathrm{GeV}^{2}$ which limits the region of our (pion) evolution. Thus the E142 results are closer to the SU(6) prediction.

\section{2}

In order to consider the pion (kaon, $\eta$-meson) contribution to the evolution of the initial constituent quarks we use the Lagrangian

$$
L=i \bar{q} \not \partial q-\bar{q} e^{i \gamma_{5}\left(\lambda^{a} \pi^{a}\right) / 2 f_{p}} M e^{i \gamma_{5}\left(\lambda^{a} \pi^{a}\right) / 2 f_{p}} q .
$$

Here $\pi^{a}$ is an octet of pseudoscalar mesons. Such a Lagrangian was obtained, for example ${ }^{3}$ in ref.[10], after spontaneous breaking of chiral symmetry in the instanton QCD vacuum, but actually it has a much wider context[11]. One may consider eq.(3) as the quark Lagrangian coupled to the pseudoscalar mesons in the presence of the $\langle 0|\bar{q} q| 0\rangle$ condensate, which provides

\footnotetext{
${ }^{2}$ An approach, which is very close to ours has been discussed recently by Ball and Forte [7] but they considered only the evolution of the Gottfried sum rule.

${ }^{3}$ In the case of $S U(2)_{F}$ flavour symmetry.
} 
rather large $(\sim 300-500 \mathrm{MeV})$ constituent quark masses $M$ for the $u, d, s$-quarks. The diagonal matrix $M$ in eq.(3) denotes the quark masses coming mainly from the $\langle 0|\bar{q} q| 0\rangle$ condensates ${ }^{4}$.

Let us make one simple comment in connection with eq.(3). It may seem that there is double counting of the degrees of freedom in eq.(3) where we use at the same time the quark and the meson fields. This is not true, however. When the chiral symmetry is unbroken the theory is invariant under chiral rotation of quarks (we neglect the small current quark masses): $q \rightarrow \exp \left(i \gamma_{5} \lambda^{a} \pi^{a}\right) q$. In this case the chiral phases of the quarks, as shown in eq.(3), play the same role as the phase of a charged scalar field $\varphi(x)=\rho(x) \exp [i \alpha(x)]$ in the case of unbroken QED. The variable $\alpha(x)$ is known not to be an appropriate degree of freedom, since it has no well-defined kinetic energy. When the chiral symmetry is broken the chiral quark phases which are nothing but pseudoscalar mesons in eq.(3), become the relevant degrees of freedom ${ }^{5}$. There is no double counting of the degrees of freedom since the definition of the chiral phases of the quark fields, entering eq.(3), is fixed by the requirement that $\langle\bar{q} q\rangle \neq 0$ while $\left\langle\bar{q} i \gamma_{5} \lambda^{a} q\right\rangle=0$. (In the case of QED the requirement corresponds to the condition that the v.e.v. of the scalar field is real). Hence, one has to add these phases back explicitly to the second term of eq.(3) in the form of the new matrix $U=e^{i \gamma_{5}\left(\lambda^{a} \pi^{a}\right) / 2 f_{p}}$, where $\lambda^{a}$ are the well known Gell-Mann matrices and the constant $f_{p}$ compensates the dimension of the pseudoscalar field $\pi^{a}\left(f_{\pi}=93 \mathrm{MeV}\right)^{6}$.

The Lagrangian of eq.(3) leads to the eight conserved non-singlet axial currents, which now explicitly contain the meson fields. In the linear approximation in $\pi^{a}$ :

$$
J_{5 \mu}^{a}=\sum_{q} \bar{q} \lambda^{a} \gamma_{5} \gamma_{\mu} q-2 f_{p} \partial_{\mu} \pi^{a}
$$

However the singlet axial current $J_{5 \mu}^{0}$ is still not conserved since we do not include here the singlet pseudoscalar meson $\eta^{\prime}$, which is much heavier than the octet of the Goldstone mesons $\pi^{a}$, due to the axial anomaly and the mixing with the $\widetilde{G}_{\mu \nu} G_{\mu \nu}$ gluon operator. Thus the model reflects the existence of Adler-Bell-Jackiw anomaly for the singlet axial current. It means that one can use the effective Lagrangian eq.(3) up to the virtualities $q^{2}$ of the order of the $\eta^{\prime}$ mass $\left(\sim 1 \mathrm{GeV}^{2}\right)$. Then $\eta^{\prime}$ should appear and the nonconservation of the singlet axial current should

\footnotetext{
${ }^{4}$ In the appendix we will discuss this point in more detail.

${ }^{5}$ The same is true for the case of the spontaneously broken QED when $\langle\rho\rangle=v$ since now the Goldstone field $a(x)=v \alpha(x)$ has the normal kinetic energy.

${ }^{6}$ There is no explicit kinetic energy terms for the pseudoscalar mesons in the Lagrangian eq.(3); they arise only from the quark loops when one integrates (3) over the quark fields. It means that the pseudoscalar mesons introduced in eq.(1) are, in fact, the composite fields.
} 
be described directly in terms of gluon fields $\left(\partial_{\mu} J_{5 \mu}^{0} \sim \widetilde{G} G\right)$.

\section{3}

In the interval of our interest $q^{2}<m_{\eta^{\prime}}^{2}$, the dimensionless quark-meson coupling constant is given by the ratio $M / f_{p}$. It should be stressed that the ratio $M^{2} / f_{p}^{2} \propto 1 / N_{c}$ is small[10] in the large $N_{c}$ (number of colours) limit. Thanks to this small parameter one can justify from the formal point of view the validity of the evolution equation in the leading $\log$ approximation. Numerically, typical values of the constant $\frac{M^{2}}{16 \pi^{2} f_{p}^{2}}$ are $<0.1-0.2$ (for our case with $N_{c}=3$ ) is small indeed.

Thus the evolution equations in the low $q^{2}$ region take the form:

$$
\begin{aligned}
\frac{\partial q_{i \downarrow}\left(x, q^{2}\right)}{\partial \ln q^{2}} & =\frac{1}{16 \pi^{2}}\left\{\sum_{j} \int_{x}^{1}\left[\Phi_{i j}^{\downarrow \uparrow}(z) q_{j \uparrow}\left(\frac{x}{z}, q^{2}\right)+\sum_{a} \Phi_{i a}(z) \pi^{a}\left(\frac{x}{z}, q^{2}\right)\right]\right. \\
& \left.\times \frac{d z}{z} \frac{M^{2}}{f_{p}^{2}}-\varphi_{i} q_{i \downarrow}\left(x, q^{2}\right)\right\} \\
\frac{\partial q_{i \uparrow}\left(x, q^{2}\right)}{\partial \ln q^{2}} & =\frac{1}{16 \pi^{2}}\left\{\sum_{j} \int_{x}^{1}\left[\Phi_{i j}^{\uparrow \downarrow}(z) q_{j \downarrow}\left(\frac{x}{z}, q^{2}\right)+\sum_{a} \Phi_{i a}(z) \pi^{a}\left(\frac{x}{z}, q^{2}\right)\right]\right. \\
& \left.\times \frac{d z}{z} \frac{M^{2}}{f_{p}^{2}}-\varphi_{i} q_{i \uparrow}\left(x, q^{2}\right)\right\}
\end{aligned}
$$

plus the same equations for $\bar{q}_{i \uparrow}$ and $\bar{q}_{i \downarrow}$ as the first two, and

$$
\begin{aligned}
\frac{\partial \pi^{a}\left(x, q^{2}\right)}{\partial \ln q^{2}} & =\frac{1}{16 \pi^{2}}\left\{\sum_{j} \int \Phi^{a j}(z)\left[q_{j \uparrow}\left(\frac{x}{z}, q^{2}\right)+q_{j \downarrow}\left(\frac{x}{z}, q^{2}\right)+\bar{q}_{j \dagger}+\bar{q}_{j \downarrow}\right]\right. \\
& \left.\times \frac{d z}{z} \frac{M^{2}}{f_{p}^{2}}-\varphi^{a} \pi_{a}\left(x, q^{2}\right)\right\} .
\end{aligned}
$$

Here the arrows indicate the helicities of the initial and final quarks. The kernels $\Phi_{i j}^{\dagger \downarrow}=\Phi_{i j}^{\downarrow \dagger}$ correspond to the emission of the pseudoscalar particles. Of course they change the helicity of the quark. Kernel $\Phi_{i j}^{\uparrow \downarrow}$ is as follows ${ }^{7}$ :

$$
\Phi_{i j}^{\uparrow \downarrow}(z)=2 N_{i j}(1-z)
$$

with

$$
N_{d d}=N_{u u}=\frac{1}{2}+h_{\eta} ; \quad N_{s s}=h_{\eta}^{\prime}
$$

\footnotetext{
${ }^{7}$ The evolution equation for the $\gamma_{5}$ theory was written for the first time in ref.[12]
} 


$$
N_{u s}=N_{s u}=N_{d s}=N_{d u}=N_{u d}=N_{d u}=1 .
$$

The terms $h_{\eta}$ and $h_{\eta}^{\prime}$ reflect the $\eta$ exchange. In the case of unbroken flavour SU(3) (without the $\eta-\eta^{\prime}$ mixing) $h_{\eta}=1 / 6$ and $h_{\eta}^{\prime}=2 / 3$ (so $2 h_{\eta}+h_{\eta}^{\prime}=1$ ). The kernels $\Phi^{a j}$ describe the emission of the $\pi, K, \eta$-mesons. They are

$$
\Phi^{a j}(z)=2 N^{a j} z,
$$

$N^{a i}=1$ for the charged $\pi^{ \pm}, K^{ \pm}$and $K^{0}, \bar{K}^{0}$ mesons;

$N^{a i}=\frac{1}{2}$ for $\pi^{0}$-meson $(i=u, d)$ and

$N^{\eta i}=h_{\eta}\left(\right.$ or $h_{\eta}^{\prime}$ ) for $\eta$-meson and $i=u, d$ (or $s$ ) quark.

Generation of quarks by the pseudoscalar parton are given by the $\Phi_{i a}$ kernels

$$
\Phi_{i a}(z)=2 N^{a i}
$$

with the same $N^{a i}$ factors as in eq.(7).

Finally the constants $\varphi_{i}$ and $\varphi^{a}$ reflect the conservation of the number of partons. After the interaction (emission of new particle) a parton which carries a momentum fraction $x$ produces a new parton with momentum fraction $x \cdot z$, but simultaneously the initial parton (with the fraction $x$ ) disappears. Thus one has to add to the r.h.s. of eqs.(5) the negative terms with

$$
\varphi_{i}=\sum_{j} \int_{0}^{1} \Phi_{i j}^{\uparrow \downarrow}(z) d z \frac{M^{2}}{f_{p}^{2}}=\sum_{j} N_{i j} \frac{M^{2}}{f_{p}^{2}}
$$

and

$$
\varphi^{a}=\sum_{i} \int_{0}^{1} \Phi_{i a}(z) d z \frac{M^{2}}{f_{p}^{2}}=\sum_{i} 2 N^{a i} \frac{M^{2}}{f_{p}^{2}} .
$$

(From the Feynman graphs point of view this contribution corresponds to the self-energy diagrams).

It will be very interesting to solve the system of eqs.(5) explicitly starting from reasonable initial distributions of the valence quarks (as was done for example for the GRV [13] structure functions of the nonpolarized nucleon) and then continue the evolution within the conventional perturbative QCD. However we do not intend to discuss the form of the initial spin dependent distributions but instead consider the combination of integrated distributions given by

$$
\Delta q=\int\left(q_{\uparrow}(x)-q_{\downarrow}(x)+\bar{q}_{\uparrow}(x)-\bar{q}_{\downarrow}(x)\right) d x .
$$

The additive quark model (AQM) SU(6) wave function leads to the initial conditions:

$$
\Delta d_{A Q M}=\Delta d_{0}=-\frac{1}{3} ; \quad \Delta u_{0}=\frac{4}{3} ; \quad \Delta s_{0}=0 .
$$


The evolution equations for $\Delta q$ are much more simple. First of all, the contributions coming from the pseudoscalar partons densities $\pi^{a}\left(x, q^{2}\right)$ cancel in the difference $q_{\uparrow}\left(x, q^{2}\right)-q_{\downarrow}\left(x, q^{2}\right)$. Next, after the integration over $d x$ the equations take the algebraic form

$$
\frac{d \Delta q_{i}}{d \ln q^{2}}=\sum_{j} \frac{a_{i j}}{16 \pi^{2}} \Delta q_{j},
$$

where

$$
a_{i j}=\frac{-M^{2}}{f_{p}^{2}} \int_{0}^{1} \Phi_{i j}^{\uparrow \downarrow}(z) d z-\delta_{i j} \varphi_{i} .
$$

The negative sign in the first term of the rhs of eq.(10) is due to the spin flip nature of the $\Phi_{i j}^{\uparrow \downarrow}$ kernel. The arrows in the 1.h.s. and in the first lines of the r.h.s. of eqs.(5) have an opposite directions. Hence, one gets the minus in front of the $\Phi_{i j}^{\uparrow \downarrow}$ term in eq.(10).

\section{4}

It is more convenient, for the numerical calculations we are going to consider below, to introduce the new effective constants: $a_{\pi}, a_{k}$ and $a_{\eta}$ corresponding to the $\pi, K$ or $\eta$-meson exchanges during the evolution eq.(9) instead of the factors $a_{i j}$.

For instance $a_{\pi}=a_{u d} / 16 \pi^{2}=\frac{1}{16 \pi^{2}} \frac{M^{2}}{f_{\pi}^{2}} \ln \frac{q_{m}^{2}}{q_{0}^{2}} \sim 0.178$. For the numerical estimate we have chosen the constituent mass $M=345 \mathrm{MeV}$ (as in ref. [10]) $f_{\pi}=93 \mathrm{MeV} ; q_{0}=M$ and $q_{m}=m_{\eta^{\prime}}=958 \mathrm{MeV}$.

To get a numerical estimation one has to diagonalize the $a_{i j}$ matrix in eq.(9) and to solve the differential equation explicitly. In the case of unbroken flavour $S U(3)_{F}$ group (when we put $a_{k}=6 a_{\eta}=3 / 2 a_{\eta}^{\prime}=a_{\pi}$ ) the eigenvectors which diagonalize the system eq.(9) are:

$$
\Delta^{0}=\Delta \Sigma=\Delta u+\Delta d+\Delta s, \quad \Delta^{3}=\Delta u-\Delta d \quad \text { and } \quad \Delta^{8}=\Delta u+\Delta d-2 \Delta s .
$$

After the evolution up to the $q^{2}=m_{\eta^{\prime}}^{2}$ the initial values $\left(\Delta_{i n}\right)$ of these eigenvectors reduce by the factors:

$$
\begin{aligned}
& \Delta^{0}=\Delta_{i n}^{0} \exp \left(-\frac{16}{3} a_{\pi}\right)=0.387 \Delta_{i n}^{0} \\
& \Delta^{3}=\Delta_{i n}^{3} \exp \left(-\frac{7}{3} a_{\pi}\right)=0.66 \Delta_{i n}^{3} \\
& \Delta^{8}=\Delta_{i n}^{8} \exp \left(-\frac{7}{3} a_{\pi}\right)=0.66 \Delta_{i n}^{8} .
\end{aligned}
$$


In other words for the polarized proton we expect

$$
\Delta u=0.789, \quad \Delta d=-0.311, \quad \Delta s=-0.091 \quad(\Delta \Sigma=0.39),
$$

while for the $\Lambda$-hyperon the analogous calculation gives:

$$
\Delta u=\Delta d=-0.09 ; \quad \Delta s=0.57 \text {. }
$$

Finally, the perturbative QCD evolution (say in the interval of $q^{2}$ from 1 to $5 \mathrm{GeV}^{2}$ ) reduces $\Delta \Sigma$ by $1.67 \%$. Thus for the nucleon

$$
\Delta u=0.787, \quad \Delta d=-0.313, \quad \Delta s=-0.093 .
$$

Coming back to eq.(11) we would like to stress that we get nontrivial evolution not only for $\Delta^{0}$ but for $\Delta^{3}$ (and $\Delta^{8}$ ) also. It does not contradict the $J_{5 \mu}^{a}$ current conservation as the conserved current eq.(4) also contains the second term $-2 f_{p} \partial_{\mu} \pi^{a}$. The divergence of the total current

$$
\partial_{\mu} J_{5 \mu}^{a}=\bar{q} \lambda^{a} \gamma_{5} k_{\mu} \gamma_{\mu} q-2 f_{p} k^{2} \pi^{a}
$$

should be equal to zero; and it does indeed vanish.

In the nucleon (or quark) brackets, the term $\left\langle\bar{q} \lambda^{a} k_{\mu} \gamma_{5} \gamma_{\mu} q\right\rangle=2 m\left\langle\bar{q} \lambda^{a} q\right\rangle$ while in the second term, where $J_{5 \mu}^{a}$ interacts with the pion $\pi^{a}$, the factor $k^{2}$ cancels the pion propagator and one gets $2 f_{p}\left\langle\bar{q} \pi^{a} q\right\rangle$. As we know, the vertex $\left\langle\bar{q} \pi^{a} q\right\rangle=m / f_{p}$ is proportional to the constituent quark mass ${ }^{8} m$. Thus the second term in the divergence of the current cancels the first one exactly. It is nothing else but the usual Goldberger-Treiman relation which reflects the axial currents conservation. Therefore the evolution of the value $\Delta^{3}=\Delta u-\Delta d$ does not violate any symmetry and is in agreement with the statement that the current $J_{5 \mu}^{a}$ is conserved.

Unfortunately the flavour $S U(3)_{F}$ group is broken. On the other hand the available interval of the logarithmic evolution is not too large $\left(\ln \frac{q_{m}^{2}}{q_{0}^{2}} \sim 2\right)$. To demonstrate that these facts do not change crucially the scale of the effect (i.e. the $\Delta q$ evolution eq. $(11,12)$ ) let us consider the first iteration of eq.(9) taking into account the smaller values of $a_{k}, a_{\eta}, a_{\eta}^{\prime}$. Due to the larger lower limit $q_{0}=m_{K}$ (or $q_{0}=m_{\eta}=549 \mathrm{MeV}$ ) we get $a_{k}=0.115$ and $a_{\eta}=0.016 ; a_{\eta}^{\prime}=0.065$.

In these terms the first iteration of eq.(9) gives

$$
\Delta u=\Delta u_{0}\left(1-2 a_{\pi}-a_{k}-2 a_{\eta}\right)-\Delta d_{0} a_{\pi}-\Delta s_{0} a_{k}
$$

\footnotetext{
${ }^{8}$ Recall that within the effective Lagrangian eq.(3) we deal with "heavy" constituent quarks (after the spontaneous breaking of the chiral symmetry).
} 


$$
\begin{aligned}
\Delta d & =\Delta d_{0}\left(1-2 a_{\pi}-a_{k}-2 a_{\eta}\right)-\Delta u_{0} a_{\pi}-\Delta s_{0} a_{k} \\
\Delta s & =\Delta s_{0}\left(1-2 a_{k}-2 a_{\eta}^{\prime}\right)-\left(\Delta u_{0}+\Delta d_{0}\right) a_{k}
\end{aligned}
$$

and thus for the proton

$$
\Delta u=0.72 ; \quad \Delta d=-0.40 ; \quad \Delta s=-0.115
$$

So the total spin $\Delta \Sigma=\Delta u+\Delta d+\Delta s$ carried by the quarks is equal to $\Delta \Sigma=0.20$.

The same calculation for the $\Lambda$-hyperon gives

$$
\Delta u=\Delta d=-a_{k}=-0.115 \quad \text { and } \quad \Delta s=0.64 \quad \text { (i.e. } \quad \Delta \Sigma=0.41 \text { ). }
$$

The estimates presented above demonstrate that the effect indeed may be large and after the emission of pseudoscalar mesons the fraction of the initial baryon spin still carried by the quarks becomes rather small (of the order of $20 \%$ ).

Let us note that after the first iteration the total quark spin $\Delta \Sigma=0.2$ is even smaller than the previous result (eq.(12)), as the second term (of the order of $a_{i j}^{2}$ ) has the opposite sign. Thus, we consider the estimate (12) as more realistic ${ }^{9}$.

5

(a)

The numbers obtained are close to experiment. The integral

$$
I^{p}=\int_{0}^{1} g_{1}(x) d x=\frac{1}{2}\left(\frac{4}{9} \Delta u+\frac{1}{9} \Delta d+\frac{1}{9} \Delta s\right)=0.152
$$

and $I^{n}=-0.031$ (i.e. for the deuteron $I^{d}=0.06$ ) while SMC [9] gives $I^{p}=0.136 \pm 0.016$. All the proton data lead to $I^{p}=0.142 \pm 0.014$ and $I^{n}=-0.028 \pm 0.006$ at $Q^{2}=5 G e V^{2}[2]$. If one uses the results of the first iteration only (eq.(16)) then $I^{p}=0.131$ and $I^{n}=-0.055$.

The SLAC data[8] taken at smaller $Q^{2} \sim 2 \mathrm{GeV}^{2}$ needed an additional comment. In the leading $\log$ evolution equation the essential values of virtuality should be much less than $Q^{2}$. Putting the usual scale $q^{2}=Q^{2} / 4$ and finishing our pseudoscalar meson emission at $q^{2}=0.5$ $\mathrm{GeV}^{2}$ one gets $a_{\pi}=0.125$. Therefore for the proton we expect instead of eq. (14),

$$
\Delta u=0.918 ; \quad \Delta d=-0.327 ; \quad \Delta s=-0.078,
$$

\footnotetext{
${ }^{9}$ We prefer to underestimate rather than to overestimate the effect. That way, our logarithmic integration starts from the largest mass in the vertex.
} 
i.e. $I^{p}=0.181$ and $I^{n}=-0.026$ which agree well with the E142 values $I^{p}=0.172 \pm 0.009$, $I^{n}=-0.022 \pm 0.011$.

It should be mentioned that the decrease in the spin carried by the quarks comes mainly from the small $x$ region. Indeed, in the case of small $x$, one has a larger interval of $z$ integration in eqs.(5) and hence a larger probability to flip the initial spin of the valence quark. This fact is also in qualitative agreement with the present experimental data on $g_{1}(x)$.

(b)

Let us demonstrate now that our simple model can also explain the Gottfried sum rule violation. After the emission of a charged pion the $u$-quark converts into the $d$ quark, changing its electrical charge. Of course it transfers this charge to the pion, but for $\pi^{+}$and $\pi^{-}$the $F_{2}$ structure functions are equal to each other $\left(F_{2}^{\pi^{+}}=F_{2}^{\pi^{-}}\right)$. So the difference

$$
\int_{0}^{1}\left[F_{2}^{p}\left(x, q^{2}\right)-F_{2}^{n}\left(x, q^{2}\right)\right] d x=\frac{1}{3} \exp \left(-2 a_{\pi}\right)=0.233
$$

to be compared with the NMC measurement of $0.236 \pm 0.016$ [14].

(c)

It is harder to check the last predictions for the $\Lambda$-hyperon. We have no $\Lambda$ target. Nevertheless the polarization of $\Lambda$ can be measured in $e^{+} e^{-}$annihilation. Thanks to the famous Gribov-Lipatov relation [15] the structure functions of deep inelastic scattering (DIS) and $e^{+} e^{-}$annihilation are closely related, $F_{e^{+} e^{-}}\left(\omega, q^{2}\right)=\frac{1}{\omega} F_{D I S}\left(\frac{1}{\omega}, q^{2}\right)$, where $\omega=(2 p q) /\left(-q^{2}\right)$. It should be stressed that the same relation is valid also for the spin dependent structure function. As we see from the original paper [16], the Altarelli-Parisi splitting kernels are the same for DIS and $e^{+} e^{-}$annihilation even for the polarized particles ${ }^{10}$. So the polarization properties of the fast $\Lambda$-hyperon produced in $e^{+} e^{-}$-annihilation should be the same as in DIS. Indeed, the vertex for polarized $\Lambda$ decay into the quark $+X$ is exactly the same as the vertex for the decay of a polarized quark into $\Lambda+X$ (in $e^{+} e^{-}$case). The only difference is the integration over the available phase space of the final system, but it is cancelled when one considers the polarizations and divides the cross section corresponding to the process with definite helicities by the unpolarized one. Thus we can expect to check the predicted spin structure of the $\Lambda$-hyperon in DIS or in $e^{+} e^{-}$-annihilation experiments.

\footnotetext{
${ }^{10}$ At least for not too small $x$ which is interesting for us.
} 
We expect that the polarization of $\Lambda$ produced in the $s$-quark jet should be equal to the $57 \%$ (see eq.(13)) of the $s$-quark polarization (in the jet thrust direction (i.e. helicity)) or $-9 \%$ for the $\Lambda$ produced in the $u$-quark current jet in DIS. Of course $57 \%$ (or $-9 \%$ ) are average values but it is better to select the leading fast $\Lambda$-hyperons (with $z>.5$ for example) in order to avoid the contributions coming from resonance decay polarization. To ensure that we are dealing with an $s$-quark jet one may detect a $K^{+}$-meson in the fragmentation (large $z$ ) region of the jet moving away in the opposite side in $e^{+} e^{-}$annihilation (or the $K^{+}$in the proton hemisphere in the case of the $s$-quark current jet from the DIS). If one detects the $\Lambda$ coming from the $u(d)$ current jet, then in DIS one has to observe the $K^{+}\left(K^{0}\right)$ meson close to the $\Lambda$ in the same jet.

In conclusion, we are encouraged by the good agreement with data. It is not evident that the model described above is crucially different from the models based on the instanton induced quark-quark t'Hooft interaction or from the bag models ${ }^{11}$ ([17], for example). However it is a very simple model and solves simultaneously two problems: the spin-crisis and Gottfried sum rule violation. Thus we hope that more detailed calculations, including the $x$-dependence of the spin dependent structure functions, may allow more precise comparison with the data.

\section{Acknowledgement}

We would like to thank M.Anselmino for many interesting discussions on all the stages of this work though we cannot suppress our disappointment with his decision not to sign this paper. One of us (M.R.) would like to acknowledge the hospitality of the RAL Theory group where part of this work was done.

\footnotetext{
${ }^{11}$ In some sense we can consider the pseudoscalar meson exchange as the low energy limit of the instanton induced interaction. At low energies the pseudoscalar mesons saturate the quark-quark interaction.
} 


\section{Appendix}

Let us discuss the possibility of introducing simultaneously the degrees of freedom which at first glance seem to be complementary to each other, namely, quarks and pions (or eight Goldstone bosons). The problem is not new, it was first discussed in[11]. The authors of ref.[11] made an assumption that the effective chiral Lagrangian can be used up to the mass scale of order $1 \mathrm{GeV}$, larger than the scale of the confinement which is crucial for the use of the quark and gluon degrees of freedom. We shall discuss the problem from a slightly different point of view.

When the chiral symmetry is unbroken the appropriate degrees of freedom are, of course, quarks and gluons, and the QCD Lagrangian (for the case of two flavours) has a standard form:

$$
\begin{gathered}
L=\bar{q} i \not D q-\bar{q} m q, \\
q=(u, d), \quad m=\left(m_{u}, m_{d}\right)
\end{gathered}
$$

where $\not D=\gamma_{\mu} D_{\mu} ; D_{\mu}$ is the covariant derivative containing the gluon field; $m_{u}, m_{d}$ are the current quark masses.

If the chiral symmetry is sponteneously broken the "chiral phases" of the quarks become the relevant degrees of freedom. We define new fields $Q(x)$ and $\pi^{a}(x)$ by

$$
q(x)=e^{-\frac{i \pi^{a}(x)}{2 f_{\pi}} \tau^{a} \gamma_{5}} Q(x)
$$

very much in the same way as we do for a Higgs doublet $\phi(x)$ for the case of weak interactions:

$$
\phi(x)=e^{i \theta^{a}(x) \tau^{a}(x)}\left(\begin{array}{c}
0 \\
\rho(x)
\end{array}\right) .
$$

We should now fix the chiral phases of $Q(x)$ in analogy to eq.A(3) where it is explicitly stated that the upper component of the doublet in the right-hand side of the equation is zero and the lower component is real. To do that for the case of eq.A(2) we recall that the chiral transformation of quarks,

$$
q(x) \rightarrow e^{i \omega^{a} \tau^{a} \gamma_{5} / 2} q(x)
$$


induces the subgroup of $O(4)$ rotations of the four-vector constructed from the quark fields

$$
J_{a}=\bar{q} i \gamma_{5} \tau^{a} q \quad, \quad J_{4}=\bar{q} q .
$$

One can check immediately that under the transformation A(4)

$$
\begin{gathered}
J_{4}=\bar{q} q \rightarrow \cos \omega(\bar{q} q)+\frac{\omega_{a}}{\omega} \sin \omega\left(\bar{q} i \gamma_{5} \tau^{a} q\right)=O_{44} \cdot J_{4}+O_{4 a} \cdot J_{a} \\
J_{a}=\bar{q} i \gamma_{5} \tau^{a} q \rightarrow\left(\delta_{a b}-2 \frac{\omega_{a} \omega_{b}}{\omega^{2}} \sin ^{2} \frac{\omega}{2}\right) \bar{q} i \gamma_{5} \tau^{b} q-\frac{\omega_{a}}{\omega} \sin \omega(\bar{q} q)= \\
=O_{a b} \cdot J_{b}+O_{a 4} \cdot J_{4} \\
\omega=\sqrt{\omega_{a}^{2}}
\end{gathered}
$$

It is obvious from these equations that three transformations $\mathrm{A}(4)$ are actually rotations in the planes $(4,1),(4,2),(4,3)$. Using these three rotations one can make three components of $J_{a}$ vanish. The chiral phases of the fields $Q(x)$ in eq.A(2) will be fixed if one imposes the conditions:

$$
\bar{Q}(x) i \gamma_{5} \tau^{a} Q(x)=0
$$

Thus in the parametrization eq.A(2), we introduce three new fields $\pi^{a}$ (pions) and impose three constraints on the fields $Q(x)$, eq.A(7).

In terms of these new degrees of freedom the Lagrangian $A(1)$ has the form:

$$
\begin{gathered}
L=\bar{Q} i \not D Q+\frac{i}{2}\left(\bar{Q} \gamma_{\mu} U^{-1} \partial_{\mu} U Q\right)+\lambda^{a}(x)\left(\bar{Q} i \gamma_{5} \tau^{a} Q\right)-\bar{Q} U^{-1 / 2} m U^{1 / 2} Q \\
U=\exp \left(-i \pi^{a} \tau^{a} \gamma_{5} / f_{\pi}\right)
\end{gathered}
$$

Here $\lambda^{a}(x)$ are the Lagrange multipliers providing the vanishing of $\bar{Q} i \gamma_{5} \tau^{a} Q=0$. The spontaneous breaking of the chiral symmetry means that the vacuum expectation value:

$$
<\bar{Q} Q>=-(250 \mathrm{MeV})^{3} \neq 0 .
$$


One should formulate this condition in terms of the fields $Q(x)$, not $q(x)$, since for the fields $Q(x)$ the equations $\mathrm{A}(7)$ fixes the chiral phases in an appropriate way (analogously, for the case of weak interactions the vacuum expectation value of $\rho(x)$ is real, $\langle\rho\rangle=v$ ). The main consequence of eq.A(9) is that the quarks $Q$ acquire a constituent mass $M$ which is the same for both flavours. After one integrates out the gluon fields in the Lagrangian A(8) the effective Lagrangian can be approximated by:

$$
L=\bar{Q} i \not \partial Q+\frac{i}{2}\left(\bar{Q} \gamma_{\mu} U^{-1} \partial_{\mu} U Q\right)-M \bar{Q} Q-\bar{Q} U^{-1 / 2} m U^{1 / 2} Q
$$

Note the difference between the constituent and the current quark mass: while the former contains no pion phases the latter does. This difference has been explicitly derived in the ref.[10]. In ref.[18] the form $\mathrm{A}(10)$ has been used to discuss the last term of this equation, i.e. the dependence of the energy on the pion phases (not their derivatives given by the second term of $\mathrm{A}(10)$ ) and the violation of the isotopic invariance.

It is sometimes useful to come back in eq.A(10) to the fields $q(x)$. We then get

$$
L=\bar{q} i \not \partial q-M\left(\bar{q} e^{i \pi^{a} \tau^{a} \gamma_{5} / f_{\pi}} q\right)-m \bar{q} q
$$

In this paper we use this last equation and neglect the current masses, putting $m=0$. 


\section{References}

[1] J. Ashman et al., Phys. Lett. B206 (1988) 364; Nucl. Phys. B328 (1989) 1.

[2] F.E. Close, R.G. Roberts, Phys. Lett. B316 (1993) 165.

G. Altarelli, G. Ridolfi, CERN-TH. 7415/94.

[3] S.L. Adler, Phys.Rev. 117 (1969) 2426;

J.S. Bell, R. Jackiw, Nuovo Cim. A51 (1969) 47.

[4] J. Kodaira, Nucl.Phys., B165 (1980) 129.

[5] E.J. Eichten, I. Hinchliffe, C. Quigg, Phys.Rev. D45 (1992) 2269.

[6] C-H. Chung, W-Y.P. Hwang Phys.Rev. D49 (1994) 2221.

[7] R.D. Ball, S. Forte Nucl. Phys. B425 (1994) 516.

[8] P.L. Anthony et al., Phys. Rev. Lett. 71 (1993) 959.

[9] B. Adeva et al., Phys. Lett. B302 (1993) 533; B320 (1994) 400;

D. Adams et al., Phys. Lett., B329 (1994) 399.

[10] D.I. Dyakonov, V.Yu. Petrov, Nucl.Phys. B272 (1986) 457.

[11] A. Manohar, H. Georgi, Nucl.Phys. B234 (1984) 189.

[12] V.N. Gribov, L.N. Lipatov, Sov.J.Nucl.Phys. 15 (1972) 438.

[13] M. Gluck, E. Reya, A. Vogt, Z.Phys. C48 (1990) 471.

[14] P. Amaudruz et al., Phys. Rev. Lett. 66 (1991) 2712.

[15] V.N. Gribov, L.N. Lipatov, Sov.J.Nucl.Phys. 15 (1972) 675.

[16] G. Altarelli, G. Parisi, Nucl.Phys. B126 (1977) 298.

[17] J. Stern, G. Clement, Nucl.Phys. A504 (1989) 621; Phys.Lett. B264 (1991) 426.

K. Steininger, W. Weise, Phys.Rev. D48 (1993) 1433.

[18] A.A. Anselm, M. Bander, Pis'ma ZhETF 59 (1994) 479. 

